\title{
On symmetric representations of groups of automorphism of bordered Klein surfaces
}

\author{
Czesław Bagiński · Grzegorz Gromadzki
}

Received: 21 September 2011 / Accepted: 9 February 2012 / Published online: 6 March 2012

(C) The Author(s) 2012. This article is published with open access at Springerlink.com

\begin{abstract}
An automorphism of a bordered compact Klein surface induces a permutation of its boundary components and we study the corresponding representations of groups of automorphisms of such surfaces in the corresponding finite symmetric groups. The principal result gives a characterization of those abstract representations of a finite group $G$ in symmetric groups which, up to the natural equivalence, proceed from compact Klein surfaces on which $G$ acts as a group of dianalytic automorphisms. We deal with such representations for actions of $G$ given by a so called smooth epimorphisms $\Lambda \rightarrow G$, where $\Lambda$ are so called nonEuclidean crystallographic groups (NEC-groups). For such data we calculate the kernels of our representations which allow, as an application, to get some results on the minimal degree of such representations for finite abstract groups.
\end{abstract}

Keywords Klein surfaces - Automorphisms of Klein surfaces · Boundary of Klein surfaces $\cdot$ NEC-groups $\cdot$ Real forms of complex algebraic curves $\cdot$ Symmetric (permutation) representation of finite groups

Mathematics Subject Classification (2000) Primary 30F; Secondary 14H

\section{Introduction}

The importance of compact connected Riemann surfaces with nontrivial groups of analytic automorphisms comes from the fact that they represent singular points in the moduli spaces of

C. Bagiński was supported by the Grant S/WI/1/2011 of Białystok University of Technology and G. Gromadzki by the Research Grant NN 201366436 of the Polish Ministry of Sciences and Higher Education.

C. Bagiński

Faculty of Computer Science, Białystok University of Technology, Wiejska 45, 15-351 Białystok, Poland e-mail: c.baginski@pb.edu.pl

G. Gromadzki $(\bowtie)$

Institute of Mathematics, University of Gdańsk, Wita Stwosza 57, 80-952 Gdańsk, Poland

e-mail: grom@mat.ug.edu.pl 
such surfaces of given genus, and the groups themselves describe the nature of such singularities in some way. On the other hand there is a well known functorial equivalence between such surfaces and smooth, irreducible, complex, projective, algebraic curves and particular role play so called symmetric Riemann surfaces which correspond to complex curves having real forms. More precisely, under this equivalence, a Riemann surface $X$ admits a symmetry $\sigma$, by which we understand an antiholomorphic involution, if and only if the corresponding curve $\mathcal{C}_{X}$ has a real form $\mathcal{C}_{X}(\sigma)$. Furthermore, two such symmetries $\sigma$ and $\tau$ define real forms $\mathcal{C}_{X}(\sigma)$ and $\mathcal{C}_{X}(\tau)$, birationally isomorphic over the field $\mathbb{R}$ of real numbers, if and only if they are conjugate in the group $\operatorname{Aut}^{ \pm}(X)$ of all, including antiholomorphic, automorphisms of $X$ and

$$
\operatorname{Aut}^{\mathbb{R}}\left(\mathcal{C}_{X}(\sigma)\right) \cong \operatorname{Cent}_{\text {Aut }^{ \pm}(X)}(\sigma),
$$

where Cent stands for the centralizer.

Now for a Riemann surface $X$ with a symmetry $\sigma$ having nonempty set of fixed points, the orbit space $X / \sigma$ is a bordered topological surface with, inherited from $X$, the natural dianalytic structure of so called Klein surface. Notice also, that each Klein surface arise from a symmetric Riemann surface in this way and a corresponding symmetry is unique up to conjugation in $\operatorname{Aut}^{ \pm}(X)$. A study of groups of dianalytic automorphisms of such surfaces is equivalent to a study of groups of birational automorphisms of real algebraic curves due to isomorphism

$$
\operatorname{Aut}(X / \sigma) \cong \operatorname{Aut}^{\mathbb{R}}\left(\mathcal{C}_{X}(\sigma)\right)
$$

and the advantage of the former is made by a counterpart of the Riemann uniformization theorem, which together with some principal facts from covering theory allows their study through a well developed combinatorial theory of non-Euclidean crystallographic groups.

Next an automorphism of a bordered Klein surface induces a permutation of its boundary components and we study the associated representations of groups of automorphisms of such surfaces in the corresponding finite symmetric groups. The principal result gives a characterization of those abstract representations of a finite group $G$ in symmetric groups which, up to the natural equivalence of symmetric representations, proceed from bordered Klein surfaces on which $\mathrm{G}$ acts as a group of dianalytic automorphisms. We deal with such representations for actions of finite groups $G$ given by a so called smooth epimorphism $\Lambda \rightarrow G$, where $\Lambda$ are so called non-Euclidean crystallographic groups. For them, we calculate the kernels of the corresponding representations which allow, as an application, to get some illustrative examples and general results on the minimal degree of such representations as well. Our results describe in such a way, a nature of groups of automorphisms of real algebraic curves.

\section{Preliminaries}

We shall use a combinatorial approach based on non-Euclidean crystallographic groups (NEC-groups in short); we send the reader to the monographs [1] and [3] for detailed exposition of the whole theory.

\subsection{Non-Euclidean crystallographic groups}

An NEC-group is a discrete and cocompact subgroup of the group $\mathcal{G}$ of isometries of the hyperbolic plane $\mathcal{H}$ including those which reverse orientation. If such a subgroup contains only orientation preserving isometries, it is called a Fuchsian group.

Macbeath [9] and Wilkie [13] associated to every NEC-group $\Lambda$ a signature $s=s(\Lambda)$, which determines its algebraic structure. It has the form 


$$
\left(g ; \pm ;\left[m_{1}, \ldots, m_{r}\right] ;\left\{C_{1}, \ldots, C_{k}\right\}\right) .
$$

Particular role, in our considerations, will play the brackets $C_{i}=\left(n_{i 1}, \ldots, n_{i s_{i}}\right)$, called the period cycles with the numbers $n_{i j} \geq 2$, called the link periods.

A group $\Lambda$ with signature (1) has the presentation with the following generators, called canonical generators:

$x_{1}, \ldots, x_{r}, e_{i}, c_{i j}, 1 \leq i \leq k, 0 \leq j \leq s_{i}$ and $a_{1}, b_{1}, \ldots, a_{h}, b_{h}$ if the sign is + or $d_{1}, \ldots, d_{h}$ otherwise,

and relators:

$$
\begin{gathered}
x_{i}^{m_{i}}, i=1, \ldots, r, c_{i j-1}^{2}, c_{i j}^{2},\left(c_{i j-1} c_{i j}\right)^{n_{i j}}, c_{i 0} e_{i}^{-1} c_{i s_{i}} e_{i}, i=1, \ldots, k, j=1, \ldots, s_{i} \text { and } \\
x_{1} \ldots x_{r} e_{1} \ldots e_{k} a_{1} b_{1} a_{1}^{-1} b_{1}^{-1} \ldots a_{h} b_{h} a_{h}^{-1} b_{h}^{-1} \text { or } x_{1} \ldots x_{r} e_{1} \ldots e_{k} d_{1}^{2} \ldots d_{h}^{2},
\end{gathered}
$$

according to whether the sign is + or - .

Generators $c_{i, j}$ are hyperbolic reflections and reflections $c_{i, j-1}, c_{i, j}$ corresponding to a link period $n_{i, j}$ are said to be consecutive or neighbouring.

Every NEC-group has associated a fundamental region, whose hyperbolic area $\mu(\Lambda)$ for an NEC-group $\Lambda$ with signature (1) is given by

$$
2 \pi\left(\varepsilon g+k-2+\sum_{i=1}^{r}\left(1-\frac{1}{m_{i}}\right)+\frac{1}{2} \sum_{i=1}^{k} \sum_{i=1}^{s_{i}}\left(1-\frac{1}{n_{i j}}\right)\right),
$$

where $\varepsilon=2$ if the sign is + and $\varepsilon=1$ otherwise. Finally, it is known that an abstract group with the above presentation can be realized as an NEC-group with the signature (1) if and only if (2) is positive and if $\Lambda^{\prime}$ is a subgroup of finite index in an NEC-group $\Lambda$ then it is an NEC-group itself and there is a Hurwitz-Riemann formula, which says that

$$
\left[\Lambda: \Lambda^{\prime}\right]=\mu\left(\Lambda^{\prime}\right) / \mu(\Lambda)
$$

\subsection{Riemann and Klein surfaces and their group of automorphisms}

A Klein surface is a compact topological surface with a dianalytic structure which, in contrast to the classical analytic structure, involve the conjugation $z \rightarrow \bar{z}$ for transition maps between charts and for the local forms of automorphisms between such surfaces. Similarly, as for Riemann surfaces, a bordered Klein surface of algebraic genus $g \geq 2$ can be represented as the orbit space $\mathcal{H} / \Gamma$ with an NEC-group $\Gamma$ having a signature $\left(g^{\prime} ; \pm ;[-] ;\{(-), . . .,(-)\}\right)$, called bordered surface NEC-group, where $k$ is the number of boundary components of $X$ and where $g=\varepsilon g^{\prime}+k-1$, with $\varepsilon=2$ if $X$ is orientable (the sign is + ) or $\varepsilon=1$ if $X$ is nonorientable (the sign is - ). Furthermore a finite group $G$ is a group of automorphisms of a surface so represented if and only if $G \cong \Lambda / \Gamma$ for some NEC group $\Lambda$ and, generally, an epimorphism $\Lambda \rightarrow G$ with the kernel being bordered surface NEC-group will be called smooth. Finally two dianalytic actions given by smooth epimorphisms $\theta: \Lambda \rightarrow G$ and $\theta^{\prime}: \Lambda^{\prime} \rightarrow G^{\prime}$ are topologically equivalent if and only if the diagram

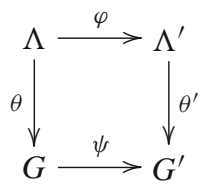

commutes for some isomorphisms $\varphi: \Lambda \rightarrow \Lambda^{\prime}$ and $\psi: G \rightarrow G^{\prime}$. 


\section{Representations of groups of automorphisms of Klein surfaces on their boundary}

Let $G$ be a group of automorphisms of a compact Klein surface $X$ with the boundary consisting of the connected components $\mathcal{O}_{1}, \ldots, \mathcal{O}_{n}$, each of which turns out to be homeomorphic to a circle. We define $\rho_{X}: G \rightarrow \mathrm{S}_{n}$ by

$$
\rho_{X}(g)(i)=j \Leftrightarrow g\left(\mathcal{O}_{i}\right)=\mathcal{O}_{j}
$$

This representation will be called geometric. With this definition we have the following

Lemma 3.1 The mapping $\rho_{X}$ is a homomorphism whose kernel is either cyclic or dihedral.

Proof If $\rho_{X}(g)(i)=i$, then $g$ induces an isometry of the circle $\mathcal{O}_{i}$. So the assertion follows from the fact that a finite group of isometries of a circle is either cyclic or dihedral, from $[5,10]$ on the extension of automorphisms of bordered Klein surfaces and from the fact that an orientation preserving automorphism of a compact Riemann surface having a non-discrete set of fixed points is trivial.

As an immediate consequence we obtain the following result of Bujalance [2] about groups of automorphisms of bordered Klein surfaces with a small number of boundary components.

Corollary 3.2 The group of automorphisms of a Klein surface having $1 \leq k \leq 4$ boundary components is solvable.

Here and throughout the remainder of the paper we shall use left hand side notation for conjugations and commutators i.e. $a^{x}=x a x^{-1}$ and $[a, x]=a x a^{-1} x^{-1}$.

Theorem 3.3 Let $\theta: \Lambda \rightarrow G$ be an epimorphism defining a group of automorphism $G$ of a Klein surface $X=\mathcal{H} / \Gamma$, where $\Gamma=\operatorname{ker} \theta$. Given a set of canonical generators of $\Lambda$, let $\mathcal{C}$ be the set of its canonical reflections. Let $\left\{c_{i}: i \in I\right\}$ be a maximal subset of $\mathcal{C}$ consisting of the pairwise non-conjugate reflections, mapped by $\theta$ into the unit. Then

$$
\operatorname{ker} \rho_{X}=\bigcap_{g \in G} \bigcap_{i \in I} g\left(\theta\left(C\left(\Lambda, c_{i}\right)\right)\right) g^{-1}
$$

Proof It is well known how to calculate the number of boundary components of a compact Klein surface $X$, in terms of $\theta$ and the signature of $\Lambda$ [3]. But here we shall need not only such quantitative results but also a qualitative description of boundary components. We shall use an approach developed in [6] and already successfully applied in [4] and [7] for example. The connected components of the boundary of $X$ are in the bijective correspondence with the reflections of $\Lambda$ which are in $\Gamma$ and which are not conjugated in $\Gamma$. On the other hand, each reflection of $\Gamma$ is conjugate to some canonical reflection from $\mathcal{C}$. So we have to look for representatives of the conjugacy classes with respect to the conjugations by the elements of $\Gamma$ in the set which, in principle comes from

$$
\left\{c^{\lambda}: c \in \mathcal{C}, \theta(c)=1, \lambda \in \Lambda\right\} .
$$

Observe however that if $c_{i} \stackrel{\Lambda}{\sim} c_{i^{\prime}}$, say $c_{i^{\prime}}=c_{i}^{\lambda}$ and $c_{i}^{\lambda^{\prime}}, c_{i^{\prime}}^{\lambda^{\prime \prime}} \in \Gamma$, then $c_{i^{\prime}}^{\lambda^{\prime \prime}}=c_{i}^{\lambda^{\prime \prime} \lambda}$. It follows that actually the canonical reflections in (4) can be runed over the set $\left\{c_{i}: i \in I\right\}$ from the statement. 
Now, given $i \in I$ we have

$$
\lambda c_{i} \lambda^{-1} \stackrel{\Gamma}{\sim} \lambda^{\prime} c_{i} \lambda^{\prime-1} \Leftrightarrow \mathrm{C}\left(\Lambda, c_{i}\right) \ni \lambda^{-1} \gamma \lambda^{\prime}=\lambda^{-1} \lambda^{\prime}\left(\lambda^{\prime-1} \gamma \lambda^{\prime}\right)
$$

for some $\gamma \in \Gamma$, which is equivalent to saying that $\lambda^{-1} \lambda^{\prime} \in \mathrm{C}\left(\Lambda, c_{i}\right) \Gamma$, as $\Gamma$ is a normal subgroup of $\Lambda$. Therefore conjugates of $c_{i}$ give rise to

$$
\left[\Lambda: \mathrm{C}\left(\Lambda, c_{i}\right) \Gamma\right]=\left[\Lambda / \Gamma: \mathrm{C}\left(\Lambda, c_{i}\right) \Gamma / \Gamma\right]=\left[G: \theta\left(\mathrm{C}\left(\Lambda, c_{i}\right)\right)\right]
$$

boundary components in $X$.

Now given $h=\theta\left(\lambda_{h}\right) \in G$ and the boundary component $\mathcal{O}$ corresponding to a reflection $c, h(\mathcal{O})$ is the boundary component corresponding to $c^{\lambda_{h}}$. Hence $h \in \operatorname{ker} \rho_{X}$ if and only if for given $i \in I$ and $\lambda \in \Lambda$, we have $\lambda_{h} \lambda c_{i} \lambda^{-1} \lambda_{h}^{-1} \stackrel{\Gamma}{\sim} \lambda c_{i} \lambda^{-1}$. This means that $\mathrm{C}\left(\Lambda, c_{i}\right) \ni$ $\lambda^{-1} \gamma \lambda_{h} \lambda=\lambda^{-1} \lambda_{h} \lambda\left(\lambda_{h} \lambda\right)^{-1} \gamma\left(\lambda_{h} \lambda\right)$ or equivalently $\lambda^{-1} \lambda_{h} \lambda \in \mathrm{C}\left(\Lambda, c_{i}\right) \Gamma$, which means that $g h g^{-1} \in \theta\left(\mathrm{C}\left(\Lambda, c_{i}\right)\right)$ for arbitrary $i \in I, g \in G$ and in turn $h \in g^{-1} \theta\left(\mathrm{C}\left(\Lambda, c_{i}\right)\right) g$.

Conversely, let

$$
h \in \bigcap_{g \in G} \bigcap_{i \in I} g\left(\theta\left(\mathrm{C}\left(\Lambda, c_{i}\right)\right)\right) g^{-1}
$$

and let $\mathcal{O}_{i}$ be a boundary component corresponding to $c_{i}^{\lambda}$ for some $\lambda \in \Lambda$. Then $h=$ $\theta(\lambda) \theta\left(\lambda_{i}\right) \theta(\lambda)^{-1}=\theta\left(\lambda \lambda_{i} \lambda^{-1}\right)$ for some $\lambda_{i} \in \Lambda$ commuting with $c_{i}$. So $h\left(\mathcal{O}_{i}\right)$ corresponds to $\left(c_{i}^{\lambda}\right)^{\lambda \lambda_{i} \lambda^{-1}}=c_{i}^{\lambda}$ and therefore $h\left(\mathcal{O}_{i}\right)=\mathcal{O}_{i}$. Thus $h \in \operatorname{ker} \rho_{X}$, which completes the proof.

Having calculated the kernel of the representation $\rho_{X}$ for the group $G$ of dianalytic automorphisms of a bordered Klein surface $X$ given by the smooth epimorphism $\theta: \Lambda \rightarrow G$, we obtain at once the following, well known and obvious.

Corollary 3.4 Given a finite group $G$, there exists a bordered Klein surface $X$ such that $G \subseteq \operatorname{Aut}(X)$ and $\rho_{X}$ is faithful.

Proof Let $g_{1}, \ldots, g_{k}$ be an arbitrary set of generators of $G$; clearly we can assume that $k \geq 2$. Let $\Lambda$ be an NEC-group with signature $(k ;+;[-] ;\{(-)\})$ and consider an epimorphism $\theta: \Lambda \rightarrow G$ for which $\theta\left(a_{i}\right)=\theta\left(b_{i}\right)=g_{i}$ and $\theta\left(e_{1}\right)=\theta\left(c_{1}\right)=1$. Then for $X=\mathcal{H} / \Gamma$, where $\Gamma=\operatorname{ker} \theta$, the representation $\rho_{X}$ is faithful by Theorem 3.3, since $\mathrm{C}\left(\Lambda, c_{1}\right)=\left\langle c_{1}, e_{1}\right\rangle$.

Remark 3.5 There is a bijection between irreducible $G$-invariant subsets of the set of boundary components of $X$ and the conjugacy classes in $\Lambda$ of canonical reflections of $\Lambda$ which are in $\Gamma$. Furthermore, the subset corresponding to the class of a reflection $c$ has $[G: \theta(\mathrm{C}(\Lambda, c))]$ elements. Indeed, (4) is the set of all reflections of $\Gamma$. As we already observed, reflections $c$ in (4) actually run over the set $\mathcal{C}^{\prime}$ of representatives of the conjugacy classes in $\Lambda$ of all canonical reflections of $\Lambda$. Observe also that given $c, c^{\prime} \in \mathcal{C}^{\prime}$ which are in $\Gamma$, their conjugations give boundary components in two disjoint $G$-invariant subsets. Finally, it is trivial that all conjugations of given $c \in \mathcal{C}^{\prime}$ are conjugate in $\Lambda$ and so the subset corresponding to $c$ is irreducible, its cardinality is found in (5).

\section{The characterization of geometric representations}

We already have shown that given a finite group there is a bordered Klein surface $X$ for which $G$ is a group of dianalytic automorphisms and $\rho_{X}$ is faithful. It should be interesting to find 
for given $G$ the minimum $n=n(G)$ so that $\rho(G) \subset \mathrm{S}_{n}$. Here we shall prove our principal result which gives necessary and sufficient conditions for an abstract permutational representation $\rho: G \rightarrow \mathrm{S}_{n}$ to be equivalent to a representation $\rho_{X}$ proceeding from a bordered Klein surface $X$ with $G$ as a group of dianalytic automorphisms.

Remark 4.1 We already know that the boundary components of $X$ correspond to the conjugacy classes in $\Gamma$ of reflections in $\Gamma$. Furthermore recall that given $g=\theta(\lambda) \in G$ and a boundary component $\mathcal{O}$, say corresponding to the reflection $c$, we have

$$
\rho_{X}(g)(\mathcal{O})=\mathcal{O}^{\prime}
$$

where $\mathcal{O}^{\prime}$ is a boundary component corresponding to $c^{\lambda}$.

A representation $\rho: G \rightarrow \mathrm{S}_{n}=\mathrm{S}(\Omega)$ of a finite group $G$ in the symmetric group on $\Omega=\{1, \ldots, n\}$ is said to be irreducible if there are no $G$-invariant proper subsets of $\Omega$. Now, given an arbitrary representation $\rho$, there is a partition $\Omega=\Omega_{1} \cup \ldots \cup \Omega_{k}$, where $\Omega_{i}$ is $G$-invariant of cardinality $n_{i}$ and it does not contain $G$-invariant subsets. In this way we obtain the decomposition $\rho=\rho_{1} \oplus \ldots \oplus \rho_{k}$ into irreducible representations $\rho_{i}$ of $G$ in $\mathrm{S}_{n_{i}}=\mathrm{S}\left(\Omega_{i}\right)$. Observe however that $\rho_{i}$ may not be faithful even if $\rho$ is faithful.

Now two representations $\rho: G \rightarrow \mathrm{S}(\Omega), \rho^{\prime}: G^{\prime} \rightarrow \mathrm{S}\left(\Omega^{\prime}\right)$ are said to be equivalent if there is an isomorphism $\alpha: G \rightarrow G^{\prime}$ and a bijection $\beta: \Omega \rightarrow \Omega^{\prime}$ such that the diagram

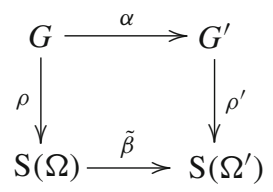

where $\tilde{\beta}(f)=\beta f \beta^{-1}$ commutes.

Observe that topologically equivalent actions give rise to equivalent representations. Finally, a representation of $G$, equivalent to the canonical representation of $G$ on the set of left cosets $G / H$ for some subgroup $H$ of $G$, is said to be regular with respect to $H$. With these definitions we have the following

Theorem 4.2 An abstract representation $\rho: G \rightarrow S_{n}$ is equivalent to the representation of a group of automorphisms of some Klein surface on its set of boundary components if and only if $\rho$ is the product of regular representations with respect to cyclic or dihedral subgroups.

Proof Let $\rho_{X}: G \rightarrow \mathrm{S}(\Omega)$ be a representation induced by the action of $G$ on the set $\Omega$ of boundary components of a Klein surface $X$ defined by a smooth epimorphism $\theta: \Lambda \rightarrow G$. Then by the Remark 3.5 we know that irreducible invariant subsets correspond to conjugacy classes of reflections of $\Lambda$ which are in $\Gamma$. Let $\Omega_{i}$ be such a subset, say of cardinality $n_{i}$, and let

$$
c_{i}^{\lambda_{1}}, \ldots, c_{i}^{\lambda_{n_{i}}}
$$


be the non-conjugate in $\Gamma$ reflections corresponding to the elements $\mathcal{O}_{1}, \ldots, \mathcal{O}_{n_{i}}$ of $\Omega_{i}$. Then, given $g=\theta(\lambda) \in G$, we have

$$
\begin{aligned}
g\left(\mathcal{O}_{j}\right)=\mathcal{O}_{k} & \Leftrightarrow c_{i}^{\lambda \lambda_{j}} \stackrel{\Gamma}{\sim} c_{i}^{\lambda_{k}} \\
& \Leftrightarrow \lambda_{k}{ }^{-1} \gamma \lambda \lambda_{j} \in \mathrm{C}\left(\Lambda, c_{i}\right) \quad \text { for some } \gamma \in \Gamma \\
& \Leftrightarrow \lambda_{k}{ }^{-1} \lambda \lambda_{j} \in \mathrm{C}\left(\Lambda, c_{i}\right) \Gamma \\
& \Leftrightarrow \theta\left(\lambda_{k}\right)^{-1} g \theta\left(\lambda_{j}\right) \in \theta\left(\mathrm{C}\left(\Lambda, c_{i}\right)\right) \\
& \Leftrightarrow g\left(\theta\left(\lambda_{j}\right) \theta\left(\mathrm{C}\left(\Lambda, c_{i}\right)\right)=\theta\left(\lambda_{k}\right) \theta\left(\mathrm{C}\left(\Lambda, c_{i}\right)\right)\right.
\end{aligned}
$$

and therefore, since, by (5), $\theta\left(\lambda_{1}\right), \ldots, \theta\left(\lambda_{n_{i}}\right)$ are representatives of all cosets in $G / \theta(\mathrm{C}$ $\left.\left(\Lambda, c_{i}\right)\right)$, we see that the action of $\rho_{X}$ on $\Omega_{i}$ is regular with respect to $\theta\left(\mathrm{C}\left(\Lambda, c_{i}\right)\right)$, which by $[11,12]$, is either cyclic or dihedral.

Conversely, assume that $\rho=\rho_{1} \oplus \ldots \oplus \rho_{k}$ is a decomposition of a representation $\rho$ into irreducible representations $\rho_{i}$ of $G$ in the symmetric group $\mathrm{S}_{m_{i}}$ on the sets $\Omega_{i}$ and suppose that $\rho_{1}, \ldots, \rho_{s}$ are regular with respect to dihedral subgroups $\mathrm{D}_{n_{i}}=\left\langle a_{i}, b_{i} \mid a_{i}^{2}, b_{i}^{2},\left(a_{i} b_{i}\right)^{n_{i}}\right\rangle$ and $\rho_{s+1}, \ldots, \rho_{k}$ are regular with respect to cyclic subgroups $Z_{n_{i}}=\left\langle a_{i}\right\rangle$. Let $m$ be the order of $a=a_{s+1} \ldots a_{k}$, let $g_{1}, \ldots, g_{l}$ be the set of generators of $G$, let $\Lambda$ be an NEC group with signature

$$
\left(l ;+;[m] ;\left\{\left(2,2, n_{1}\right), \ldots,\left(2,2, n_{s}\right),(-), \stackrel{k-s}{.},(-)\right\}\right)
$$

and let $\theta: \Lambda \rightarrow G$ be an epimorphism defined by the assignment

$$
\begin{gathered}
\theta\left(a_{i}\right)=\theta\left(b_{i}\right)=g_{i} \quad \text { for } i=1, \ldots, l, \\
\theta\left(c_{i 0}\right)=\theta\left(c_{i 3}\right)=a_{i}, \theta\left(c_{i 1}\right)=1, \theta\left(c_{i 2}\right)=b_{i}, \theta\left(e_{i}\right)=1 \text { for } i=1, \ldots s, \\
\theta\left(c_{i 0}\right)=1, \theta\left(e_{i}\right)=a_{i} \text { for } i=s+1, \ldots, k, \text { and } \theta\left(x_{1}\right)=a^{-1} .
\end{gathered}
$$

Then for $X=\mathcal{H} / \Gamma$, where $\Gamma=\operatorname{ker} \theta$ we have a representation $\rho_{X}: G \rightarrow \mathrm{S}_{n}$, where $n=n_{1}+\cdots+n_{k}$, equivalent to $\rho$.

\section{On the degree of geometrical representations of finite groups}

Let $G=\Lambda / \Gamma$, where $\Lambda$ is an NEC group, $\Gamma$ is a surface NEC-group and let $\theta: \Lambda \rightarrow G$ be the corresponding smooth epimorphism. We know that given a finite group $G$ there is a bordered Klein surface $X$ with $k$ boundary components for which $\rho_{X}: G \rightarrow \mathrm{S}_{k}$ is faithful and a natural problem considered here is to find the minimal $k$ in such situation.

5.1 A general theorem

Theorem 5.1 The minimum degree $k(G)$ of a geometrical representation of a finite group $G$ is a minimal value of

$$
\frac{1}{2}\left(\frac{N}{\left|d_{1} d_{1}^{\prime}\right|}+\cdots+\frac{N}{\left|d_{s} d_{s}^{\prime}\right|}\right)+\frac{N}{\left|a_{s+1}\right|}+\cdots+\frac{N}{\left|a_{k}\right|},
$$


where $|x|$ denotes the order of $x, s \leq k$ run over all nonnegative integers, $a_{i}$ are arbitrary elements and $d_{i}^{\prime}, d_{i}$ are arbitrary involutions for which

$$
\left(\bigcap_{g \in G} \bigcap_{j=1}^{s} g\left\langle d_{j}, d_{j}^{\prime}\right\rangle g^{-1}\right) \bigcap\left(\bigcap_{g \in G} \bigcap_{i=s+1}^{k} g\left\langle a_{i}\right\rangle g^{-1}\right)=1
$$

Proof Let $G=\Lambda / \Gamma$. Observe, that for a reflection $c$ corresponding to an empty period cycle we have $\mathrm{C}(\Lambda, c)=\langle e, c\rangle$ while for a triple consecutive reflections $c^{\prime}, c, c^{\prime \prime}$ corresponding to two consecutive link periods equal to $2, \mathrm{C}(\Lambda, c)=\langle c\rangle \oplus\left\langle c^{\prime}, c^{\prime \prime}\right\rangle$. On the other hand, each boundary component of $X=\mathcal{H} / \Gamma$ correspond to a conjugate of a canonical reflection $c$ of $\Lambda$ which is in $\Gamma$. Furthermore, if such a reflection corresponds to an empty period cycle with connecting generator $e$, then it gives rise to $|G| /|\theta(e)|$ boundary components of $X$ by (5), while if it corresponds to a nonempty period cycle, then for the neighbouring reflections $c^{\prime}, c^{\prime \prime}$, the products $c^{\prime} c$ and $c c^{\prime \prime}$ have orders 2 and $c$ giving rise to $|G| / 2\left|\theta\left(c^{\prime}\right) \theta\left(c^{\prime \prime}\right)\right|$ boundary components in $X$ by (5) again. There are no boundary components of $X$ which arise in any other way and so the minimal value of (8) give a lower bound for $k(G)$ in virtue of Theorem 3.3 .

On the other hand having arbitrary elements $a_{i}$ and arbitrary involutions $d_{i}^{\prime}, d_{i}^{\prime \prime}$ for which (8) is minimal, let $g_{1}, \ldots, g_{l}$ be an arbitrary set of generators for $G$. Then an epimorphism constructed in the second part of Theorem 4.2 gives a Klein surface $X$ with a group of automorphisms $G$ for which $\rho_{X}$ is a geometrical permutation representation of $G$ of the minimal degree.

\subsection{Groups for which $k(G)=|G|$}

It is obvious that $k(G) \leq|G|$ and here we shall look for the groups for which $k(G)=|G|$. We start with rather obvious

Example 5.2 Let $G=\langle g\rangle=\mathrm{Z}_{N}$, be a cyclic group of order $N$, where $N=p_{1}^{\alpha_{1}} \ldots p_{r}^{\alpha_{r}}$ for different primes $p_{1}, \ldots, p_{r}$ and $r>1$. Let $N_{i}=N / p_{i}^{\alpha_{i}}, i=1, \ldots, r$. The subgroup $G_{i}=\left\langle g^{p_{i}^{\alpha_{i}}}\right\rangle$ has index $p_{i}^{\alpha_{i}}$ in $G$. Moreover

$$
\bigcap_{1 \leq i \leq r} G_{i}=1
$$

Hence, there exists a geometrical representation of degree $p_{1}^{\alpha_{1}}+\cdots+p_{r}^{\alpha_{r}}$ which is the minimum degree of a faithful permutation representation for a cyclic group of order $N$. If $r=1$ then obviously the minimum degree of a geometrical representation is equal to $N$.

Example 5.3 Let $\mathrm{D}_{N}$ be a dihedral group of order $2 N$, where $N=p_{1}^{\alpha_{1}} \ldots p_{r}^{\alpha_{r}}, p_{1}, \ldots, p_{r}$ are different primes and $r>1$. Let $G$ be a cyclic subgroup of $\mathrm{D}_{N}$ of order $N$. Every element of $G$ is a product of two involutions, so for a subgroup $G_{i}, i=1, \ldots, r$, from the previous example, there exist involutions $d_{i}, d_{i}^{\prime}$ such that $\left|\left\langle d_{i}, d_{i}^{\prime}\right\rangle: G_{i}\right|=2$. Similarly as in Example 5.2 we see that

$$
G \cap \bigcap_{1 \leq i \leq r}\left\langle d_{i}, d_{i}^{\prime}\right\rangle=1
$$


The maximal subgroup of $\mathrm{D}_{N}$ which has trivial intersection with $G$ has order 2 and so it is generated by an involution. No element outside $G$ is central, so

$$
\bigcap_{g \in G} \bigcap_{1 \leq i \leq r} g^{-1}\left\langle d_{i}, d_{i}^{\prime}\right\rangle g=1 .
$$

Now suppose that $N$ is a power of a prime, $N>2$. Then for a noncentral involution $d \in \mathrm{D}_{N}$ we have

$$
\bigcap_{g \in G} g^{-1}\langle d\rangle g=1 .
$$

Hence the minimum degree of a faithful geometrical representation is equal to $\left|\mathrm{D}_{N}\right| / 2=N$ and is the same as the minimum degree of a faithful permutation representation.

Example 5.4 Let $\mathrm{Q}_{2^{n}}$ be a generalized quaternion group of order $2^{n+1}$ :

$$
\mathrm{Q}_{2^{n}}=\left\langle x, y \mid x^{2^{n}}=1, y^{2}=x^{2^{n-1}} y^{-1} x y=x^{-1}\right\rangle .
$$

It is well known that every nontrivial subgroup of $\mathrm{Q}_{2^{n}}$ contains a subgroup $\left\langle x^{2^{n-1}}\right\rangle$. Therefore we obtain the minimal value of (8) for $r=1$ and $a_{1}=1$. Hence the minimum degree of a faithful geometrical representation is equal to $2^{n+1}$.

Proposition 5.5 If $G$ is a finite group then $k(G)=|G|$ if and only if $G$ is one of the following groups:

(a) a cyclic group of order a power of a prime,

(b) a generalized quaternion group,

(c) an elementary abelian group of order $2^{n}$.

Proof The implication $\Leftarrow$ follows from the previous examples and we shall prove the converse. If $|G|$ is divisible by two different primes $p$ and $q$ then it contains two cyclic subgroups of orders $p$ and $q$ respectively. Since they have trivial intersection, $k(G) \leq$ $|G| / p+|G| / q<|G|$. Thus $G$ is a $p$-group. If every two nontrivial subgroups of $|G|$ have nontrivial intersection then either $G$ is cyclic or $p=2$ and $G$ is a generalized quaternion group (see for instance [8], Satz 5.3.7). Suppose $G$ has two subgroups $\left\langle a_{1}\right\rangle$ and $\left\langle a_{2}\right\rangle$ of order $p^{m}$ and $p^{k}$ respectively. Then $k(G) \leq|G|\left(1 / p^{m}+1 / p^{k}\right)$. If the right hand side of this inequality is equal to $|G|$, then $p=2$ and $m=k=1$, which means that $G$ is elementary abelian of order $2^{n}$, for some $n$.

It is known that if we put $n=2$ in the last item of the above proposition we get the description of groups $G$ for which the minimum degree of a faithful permutation representation is equal to $|G|$.

5.3 On $k(G)$ for finite simple groups at large

If $G$ is a finite simple group then the sum from Theorem 5.1 has obviously only one component coming from a cyclic or dihedral subgroup of maximal order.

Lemma 5.6 An arbitrary maximal subgroup of a finite simple group is not cyclic. 
Proof Suppose that a maximal subgroup $M$ of a finite simple group $G$ is cyclic. Then obviously $N_{G}(M)=M$ and for every $g \in G \backslash M$ we have $g^{-1} M g \cap M=1$. Otherwise $g^{-1} M g \cap M$ would be a nontrivial central subgroup in $\left\langle g^{-1} M g, M\right\rangle=G$ which contradicts the simplicity of $G$. Hence, by Frobenius theorem, $M$ has a nontrivial normal complement which again gives a contradiction.

On the other hand there exist infinitely many finite simple groups containing maximal subgroups which are dihedral. But also in this case $k(G)$ does not need to be equal to the minimal degree of a permutation representation of $G$.

Lemma 5.7 Let $p$ be a prime, $p>3$, and let $G=\operatorname{PSL}(2, p)$. Every maximal subgroup $M$ of $G$ is isomorphic to one of the following groups:

(a) a dihedral group of order $p-1$ or $p+1$,

(b) a semidirect product of a Sylow p-subgroup of $G$ and a cyclic group of order $(p-1) / 2$,

(c) $A_{4}, A_{5}$ or $S_{4}$-the alternating group of degree 4 or 5 or the symmetric group of degree 4.

Example 5.8 If $p$ is a prime, $p>3$, then $k(\operatorname{PSL}(2, p))$ is bigger than the minimum degree of a permutation representation of $\operatorname{PSL}(2, p)$ because maximal subgroups of the biggest order are those described in the items $(b)$ and $(c)$. It is immediately seen that the minimal degree of a faithful permutation representation of $\operatorname{PSL}(2, p)(p>11)$ is equal to $p+1$, when $k(\operatorname{PSL}(2, p))=p(p-1) / 2$.

The minimum degree of faithful permutation representations of finite simple groups were determined in a series of papers by several authors. For many reasons it would be interesting to describe $k(G)$ for these groups, which however is not an easy matter and perhaps is a good task for further and more detailed study and a sequel is planned by the authors. We finish this paper with an example of alternating groups which indicates some difficulties in determining the precise value of $k(G)$.

Example 5.9 We start with the symmetric group $\mathrm{S}_{n}$ of degree $n$, where the minimum degree of a faithful permutation representation is obviously equal to $n$. Let $s$ be the maximal order of elements of $\mathrm{S}_{n}$, which is the maximum of the least common multiple of numbers $n_{1}, n_{2}, \ldots, n_{k}$ such that $n_{1}+\cdots+n_{k} \leq n$. Since each cyclic subgroup of $\mathrm{S}_{n}$ is contained in a subgroup which is dihedral we have $k(G)=n ! / 2 s$ which, for instance, give $k\left(\mathrm{~S}_{5}\right)=10$ and $k\left(\mathrm{~S}_{8}\right)=8 ! / 30=1344$. The case of alternating groups $\mathrm{A}_{n}$ is a little bit more involved. Here also the minimum degree of a permutation representation is equal to $n$ but now a cyclic subgroup of the maximal order may not be contained in a dihedral subgroup which makes the problem nontrivial. For instance the maximal order of elements of $A_{7}$ is 7 , but the normalizer of a subgroup of order 7 in $A_{7}$, has order 21 (there is not a subgroup of order 14) and it is easily seen that $k\left(\mathrm{~A}_{7}\right)=(7 ! / 2) /(2 \cdot 6)=210$ as $\mathrm{A}_{7}$ contains a dihedral subgroup of order 12. The maximal order of elements of $A_{8}$ is 15 , that is the same as for $S_{8}$, but there is not a dihedral subgroup of order $\geq 15$. Hence $k\left(\mathrm{~A}_{8}\right)=k\left(\mathrm{~S}_{8}\right)$. In the general case $\mathrm{A}_{n}, n>5$ one can write that $k(G) \geq n ! / 4 s$, where $s$ is the maximal order of an element of $\mathrm{A}_{n}$.

Acknowledgments The authors are very grateful to both referees for their accurate and helpful suggestions and comments concerning submitted versions of the paper which allowed us to improve it essentially.

Open Access This article is distributed under the terms of the Creative Commons Attribution License which permits any use, distribution, and reproduction in any medium, provided the original author(s) and the source are credited. 


\section{References}

1. Alling, N.L., Greenleaf, N.: Foundations of the theory of Klein surfaces. Lecture Notes in Mathematics, vol. 219. Springer, Berlin (1971)

2. Bujalance, E.: On the structure of the automorphism group of a compact Klein surface with $N$ boundary components $(1 \leq N \leq 4)$. Math. Ann. 270, 29-34 (1985)

3. Bujalance, E., Etayo, J.J., Gamboa, J.M., Gromadzki, G.: Automorphism groups of compact bordered Klein surfaces, a combinatorial approach. Lecture Notes in Mathematics, vol. 1439. Springer, Berlin (1990)

4. Bujalance, E., Gromadzki, G.: On automorphisms of unbordered Klein surfaces with invariant discrete subsets. Osaka J. Math. (2012, in press)

5. Greenleaf, N., May, C.L.: Bordered Klein surfaces with maximal symmetry. Trans. Am. Math. Soc. 274, 265-283 (1982)

6. Gromadzki, G.: On a Harnack-Natanzon theorem for the family of real forms of Riemann surfaces. J. Pure Appl. Algebra 121, 253-269 (1997)

7. Gromadzki, G.: On ovals on Riemann surfaces. Revista Matematica Iberoamericana 16(3), 515-527 (2000)

8. Kurzweil, H., Stellmacher, B.: Theorie der endlichen Gruppen. Springer, Berlin (1998)

9. Macbeath, A.M.: The classification of non-Euclidean crystallographic groups. Can. J. Math. 19, 1192-1205 (1967)

10. Oikawa, K.: Notes on conformal mappings of a Riemann surface onto itself. Kodai math. Sem. Rep. 8(suppl.), 23-30, 115-116 (1956)

11. Singerman, D.: Non-Euclidean crystallographic groups and Riemann surfaces. PhD thesis, University of Birmingham (1969)

12. Singerman, D.: On the structure of non-Euclidean crystallographic groups. Proc. Camb. Philos. Soc. 76, 233-240 (1974)

13. Wilkie, D.: On non-Euclidean crystallographic groups. Math. Z. 97, 87-102 (1966) 